\title{
Anabases
}

ANABASES Traditions et réceptions de l'Antiquité

$30 \mid 2019$

Varia

\section{Ovide en classe, ou un auteur en éclats}

\section{Martine Furno}

\section{(2) OpenEdition}

Journals

Édition électronique

URL : https://journals.openedition.org/anabases/9957

DOI : 10.4000/anabases.9957

ISSN : 2256-9421

\section{Éditeur}

E.R.A.S.M.E.

\section{Édition imprimée}

Date de publication : 21 octobre 2019

Pagination : 127-142

ISSN : 1774-4296

\section{Référence électronique}

Martine Furno, "Ovide en classe, ou un auteur en éclats », Anabases [En ligne], 30 | 2019, mis en ligne le 21 octobre 2021, consulté le 08 novembre 2021. URL : http://journals.openedition.org/anabases/ 9957 ; DOI : https://doi.org/10.4000/anabases.9957 

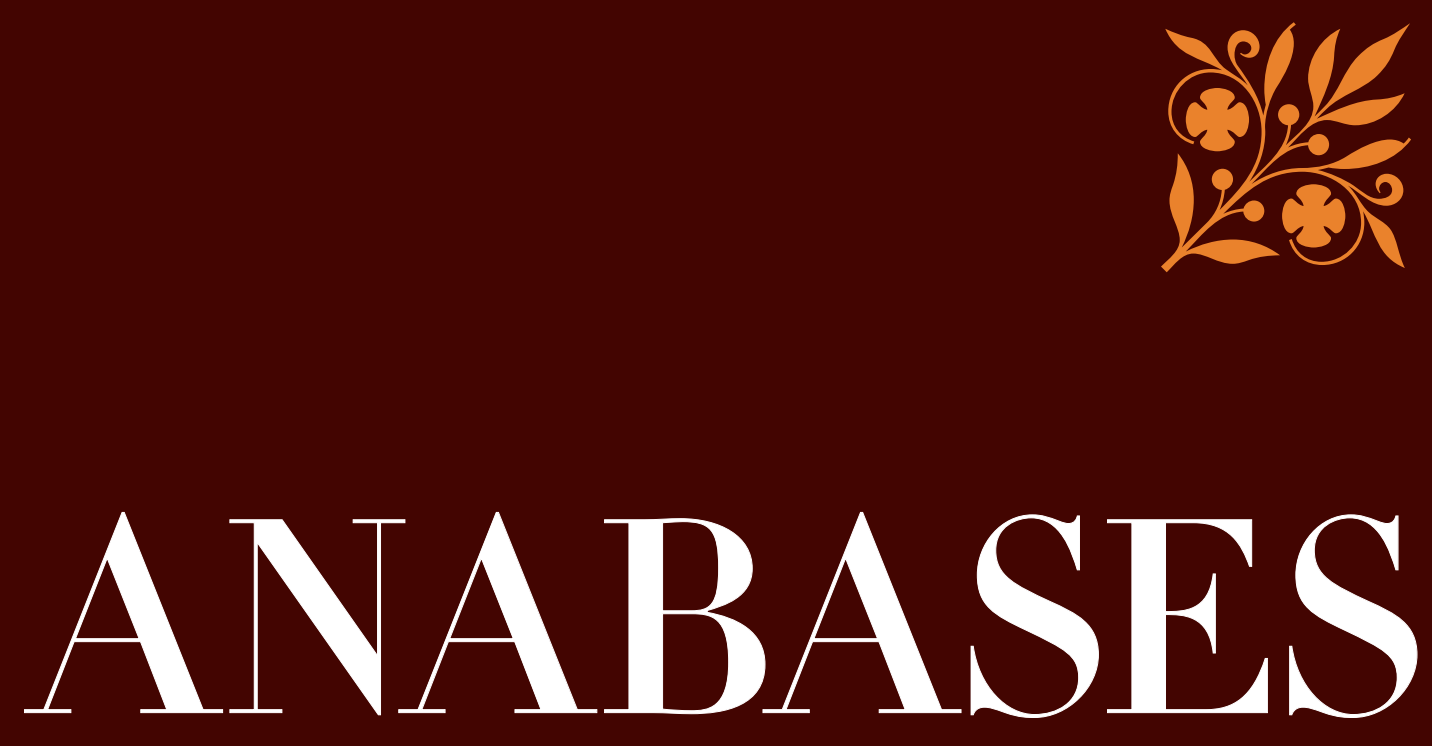

Traditions et Réceptions de l'Antiquité

\section{$\mathrm{N} \times 30$ \\ 2019}

Le sanctuaire de Gournay-sur-Aronde Le pindarisme et l'archéologie musicale Hélène en Égypte Perpétuer Ovide aux $X I V^{e}-X V I I I^{e}$ siècles II classico si fa pop Relire Marcel Detienne Freud à Pompéi 
ANABASES

Traditions et Réceptions de l'Antiquité

Revue de l'équipe de recherche E.R.A.S.M.E.

Université Toulouse-Jean Jaurès (UT2J)

Anabases dispose d'un Comité de lecture international. Chaque article envoyé à la rédaction est soumis, une fois anonymisé, à l'expertise de deux spécialistes qui rendent un rapport écrit. Les deux rapports anonymisés sont transmis à l'auteur qui tient compte des observations en vue de la publication.

\section{Comité SCIENTIFIQue}

Germaine Aujac (université Toulouse-Jean Jaurès : histoire de la géographie et des sciences antiques)

Florence Bouchet (université Toulouse-Jean Jaurès : littérature médiévale)

Hinnerk Brunns (CNRS : histoire économique et sociale ancienne et contemporaine)

Paulo Butti de Lima (université de Bari : historiographie et réception de l'Antiquité)

Luciano CANFora (université de Bari : littérature et histoire anciennes, historiographie)

Giovanna Ceserani (Stanford University : histoire intellectuelle et historiographie de la tradition classique)

Temístocles Cezar (université de Porto Alegre : historiographie moderne)

Serafina Сuомо (University of London, Birkbeck College : histoire des mathématiques et des sciences)

Paul Demont (université de Paris Sorbonne : philologie grecque et héritage classique)

Marie-Laurence Desclos (université de Grenoble II : philosophie de l'Antiquité)

Olivier Devillers (université de Bordeaux 3 - Michel-de-Montaigne : littérature et historiographie latines)

Andrea Giardina (Istituto italiano di scienze umane : histoire du monde romain et de ses réceptions)

Ève Gran-Aymerich (AIBL : histoire de l'archéologie et des transferts culturels)

François Hartog (eHess : historiographie ancienne et moderne)

Geneviève Hoffmann (université de Picardie : histoire des mondes grecs)

Christian JACOB (CNRS/EHESS : histoire comparée et épistémologie des savoirs)

Suzanne Marchand (Louisiana State University : histoire du classicisme et de l'orientalisme)

Wilfried NIPPEL (Humboldt Universität Berlin : histoire et historiographie de l'Antiquité)

Sylvie Pitria (université de Paris I-Panthéon Sorbonne : histoire et historiographie du monde romain)

Stéphane Ratтi (université de Franche-Comté - Besançon : philologie et héritage latin)

Comité de RÉdACtion

Clément Bertau-Courbières, Corinne Bonnet, Laurent bricault, Clément Bur, Adeline Grand-Clément, Anne-Hélène Klinger-Dollé, Véronique Krings, Thibaud Lanfranchi, Pascal Payen, Grégory Reimond, Sarah Rey, Catherine Valenti, Noémie VillacèQue

ÉditeUr RESPONSABLE

Clément Bur

Éditrice ADJOINTE

Catherine VALENTI

Sites Web

http://plh.univ-tlse2.fr

Revues.org : http://anabases.revues.org

Aвonnement et vente aU numéro

Éditions De Boccard - 4, rue de Lanneau - 75005 Paris

info@deboccard.com - www.deboccard.com

Tél. : 0033/(0)143260037 - Fax : 0033/(0)143548583 




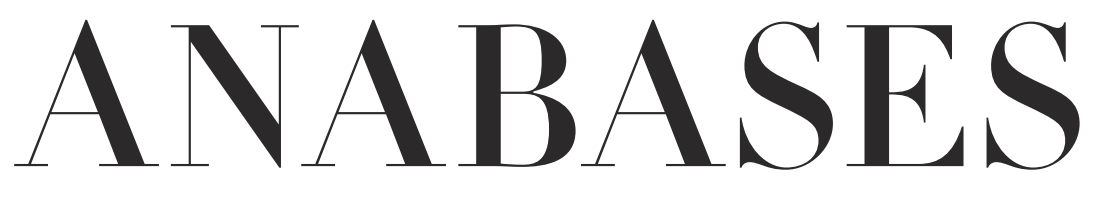

Traditions et Réceptions de l'Antiquité

$$
\begin{aligned}
& N \circ 30 \\
& 2019
\end{aligned}
$$

\section{E.R.A.S.M.E.}

Université Toulouse - Jean Jaurès 



\section{Sommaire}

ํo30 - 2019

\section{Historiographie et identités culturelles}

\section{Carole Quatrelivre}

Le sanctuaire gaulois de Gournay-sur-Aronde (Oise).

Retour sur une découverte exceptionnelle des années I970 . . . . . . . . II

\section{Traditions du patrimoine antique}

Christophe Corbier

Le pindarisme et l'archéologie musicale : style, valeur et authenticité

de la première Pythique à l'époque moderne . . . . . . . . . . . . . . .

Arnaud AmiLIEN

Hélène en Égypte : Hérodote en dialogue avec l’épopée . . . . . . . . . .

\section{Archéologie des savoirs}

Sébastien Cazalas

Au jardin des exempla. Rhétorique et stratégie de l'exemplum antique

dans l'œuvre politique de Jean Juvénal des Ursins (I388-ı473) . . . . . . 7 7

Dossier - Perpétuer Ovide : aspects moraux, éditoriaux,

linguistiques et culturels $\left(\mathrm{XIV}^{\mathrm{e}}-\mathrm{XVIII}^{\mathrm{e}} \mathrm{s}\right.$.)

Francesca Dell'Oro

Introduction $\ldots \ldots \ldots \ldots$. . . . . . . . . . . . . . 89

Hélène Casanova-Robin

L’audace châtiée : Phaéton, Actéon et Icare dans la tradition latine jusqu'à la Renaissance, tours et détours d'un symbolisme . . . . . . . . 9 93 
Dylan Bovet

Le commentaire latin des Métamorphoses d'Ovide : pratiques humanistes et évolutions de Regius-Micyllus (I543) à Burmann-Heinsius (I727) . . . . III

Martine FurNo

Ovide en classe, ou un auteur en éclats . . . . . . . . . . . . . I27

Basil NELIS

D’un Ovide chrétien à un Ovide burlesque, du Moyen Âge au Grand Siècle : continuités et changements dans la traduction et dans l'illustration des Métamorphoses perçus à travers deux éditions du xvII ${ }^{\mathrm{e}}$ siècle . . . . . . . I I43

Olivier ThÉvenAz

Épilogue ....................... I6

\section{Actualités et débats}

Tiphaine-Annabelle BEsnard

2019 : l'année pop des musées d'archéologie. Retour sur l'exposition

romaine Il classico sifa pop. Di scavi, copie e altripasticci . . . . . . . . . . I I7I

\section{Lire, relire la bibliothèque des sciences de l'Antiquité}

Jean-Pierre Albert

Le premier Detienne : une relecture de

“ La notion mythique d"A $\lambda \hat{n} \theta \varepsilon 1 \alpha$ » $\left(R E G\right.$, I96o, p. 27-35) . . . . . . . . . . $\quad{ }_{779}$

Marcel Detienne

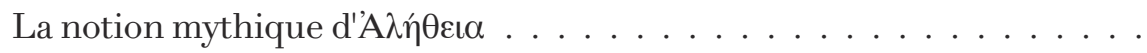

\section{Ateliers de l'histoire}

Antiquités numériques (coordonné par Elodie Guillon) ( $n^{\circ} 1$ )

Élodie GuILlon

Introduction

Jaime Alvar

Le projet EPIDI : Epítetos divinos.

Experiencia religiosa y relaciones de poder en Hispania . . . . . . . . . . . I98 Les mots de l'Antiquité (coordonné par Magali Soulatges) ( $n^{\circ} 10$ )

Jack Thомаs

L’Antiquité dans les toponymes de l'État de New York . . . . . . . . . . . 202

Actualité du théâtre (coordonné par Malika Bastin-Hammou) ( $\left.n^{\circ} 4\right)$

Mathieu FERrand

“ Avons-nous perdu le Soleil ? / Ou l'avons-nous chassé ? » 
Thyeste de Sénèque, traduit par Florence Dupont.

Mise en scène de Thomas Jolly (Avignon, 20I8) . . . . . . . . . . . . . 2 2I4

Voyages et Voyageurs (coordonné par Véronique Krings) ( $n^{\circ} 11$ )

Claude AzIzA

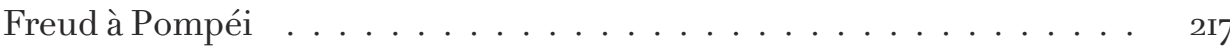

\section{Comptes rendus}

Lucile Arnoux-Farnoux et Polina Kosmadaki (dir.)

Le double voyage : Paris-Athènes (Igrg-Ig39) (Catherine Valenti) . . . . . . 225

Sandra Boehringer et Daniele Lorenzini (dir.)

Foucault, la sexualité, l'Antiquité (Jan Nelis) . . . . . . . . . . . . . . . . 226

Corinne Bonnet, Nicole Belayche, Marlène Albert Llorca,

Alexis Avdeeff, Francesco Massa, Iwo Slobodzianex (dir.)

Puissances divines à l'épreuve du comparatisme. Constructions,

variations et réseaux relationnels (Geneviève Hoffmann) . . . . . . . . . . 228

Shane ButLer (éd.)

Deep Classics, Rethinking Classical Reception (Jan Nelis) . . . . . . . . . . 23o

Zeynep ÇELIK

About Antiquities: Politics of Archaeology in the Ottoman Empire (Jorge Elices Ocón) . . . . . . . . . . . . . . . . . 231

Xavier Deru et Germaine Leman-Delerive (éd),

Franz Cumont, Comment la Belgique fut romanisée (Vivien Barrière) . . . 234

Olivier Devillers, Breno Battistin Sebastiani (éd.)

Sources et modèles des historiens anciens (Arnaud Saura-Ziegelmeyer) . . $\quad 235$

Mara Fazio, Pierre Frantz et Vincenzo De Santis (dir.)

Les Arts du spectacle et la référence antique dans le théâtre

européen (I760-I830) (Arnaud Saura-Ziegelmeyer) . . . . . . . . . . . . . . 237

Jérémy Guedu et Barbara Meazzi (dir.)

La culture fasciste entre latinité et méditerranéité (I880-1940), in Cahiers de la Méditerranée 95, (Andrea Avalli) . . . . . . . . . . . . . . . 239

Marie-Laurence HAAck (éd.), avec la collaboration de Martin MiLler, Les Étrusques au temps du fascisme et du nazisme (Jan Nelis) . . . . . . . . . 24I

Arlene Holmes-Henderson, Steven Hunt et Mai Musié (éd.)

Forward with Classics. Classical Languages in Schools and Communities

(Charlotte Tournier) . . . . . . . . . . . . . . . . . . . . . . .

Daniel Jew, Robin Osborne et Michael Scotт (éd.)

M. I. Finley. An Ancient Historian and his Impact (Hinnerk Bruhns) . . . . $\quad 244$ 
Kostas Kalimtzis,

An inquiry into the philosophical concept of scholê.

Leisure as a Political End (Florent Rouzade) . . . . . . . . . . . . . . . . . 246

Anne-Hélène KLINGER-Dollé

Le De sensu de Charles de Bovelles. Conception philosophique

des sens et figuration de la pensée. Suivi du texte latin du De sensu,

traduit et annoté (Laure Hermand-Schebat) . . . . . . . . . . . . . 248

Egidia Occhipinti

The Hellenica Oxyrhynchia and Historiography:

New Research Perspectives (Anne de Cremoux) . . . . . . . . . . . . . . . . 249

Laurent OLIVIER (dir.)

La mémoire et le temps. L'ouvre transdisciplinaire

d'Henri Hubert (I872-I927) (Sarah Rey) . . . . . . . . . . . . . . . 25I

Gabriella Pinonti et Corinne Bonnet (dir.),

Les dieux d'Homère. Polythéisme et poésie en Grèce ancienne,

Kernos, Supplément 3r. (Sandya Sistac) . . . . . . . . . . . . . . . 253

Rabun TAYLoR, Katherine W. Rinne et S. Kostof

Rome. An Urban History from Antiquity to the Present

(Cyrielle Landrea) . . . . . . . . . . . . . . . . . . . 255

Wyger Velema et Arthur Weststeisn (éd.)

Ancient Models in the Early Modern Republican Imagination

(Paulo Butti de Lima) . . . . . . . . . . . . . . . . . . . 256

Philip WaLsh (éd.)

Brill's Companion to the Reception of Aristophanes

(Malika Bastin-Hammou) . . . . . . . . . . . . . . .

Richard WARren

Art Nouveau and the Classical Tradition (Lucien Calvié) . . . . . . . . . 26o

Jesse Weiner, Benjamin Eldon Stevens et Brett M. Rogers (éd.)

Frankenstein and Its Classics. The Modern Prometheus

from Antiquity to Science Fiction (Mathieu Scapin) . . . . . . . . . . . . . 26r

Nigel G. WiLson

From Byzantium to Italy. Greek Studies in the Italian Renaissance,

deuxième édition (Luigi-Alberto Sanchi) . . . . . . . . . . . . . . 262

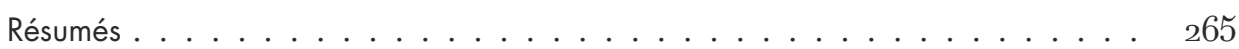

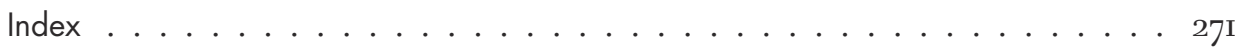




\section{Dossier dirigé par Francesca Dell'Oro et Olivier Thévenaz}

Perpétuer Ovide : aspects moraux, éditoriaux, linguistiques et culturels $\left(\mathrm{XIV}^{\mathrm{e}}-\mathrm{XVIII}{ }^{\mathrm{e}} \mathrm{s}\right.$.) 



\title{
Ovide en classe, ou un auteur en éclats
}

\author{
Martine FurNo
}

$\mathrm{O}$ vide est un auteur paradoxal dans I'histoire de la transmission de la culture latine. Sa lecture a fasciné le Moyen Âge dès le xir siècle, au point d'en faire après Virgile l'auteur le plus lu à cette époque par les lettrés, en latin comme en langue vernaculaire ; mais il reste un auteur connu par certaines de ses œuvres, non par l'ensemble de celles-ci.

Cette caractéristique tient sans doute à la place donnée à cet auteur dans le cursus scolaire. Poète léger voire immoral dans certaines élégies, classique un peu moralisateur dans les textes de l'exil et certains passages des Métamorphoses, historien utile pour documenter l'histoire de Rome dans les Fastes : autant de facettes difficiles à présenter en classe. Les écoles ont souvent préféré à Ovide les contours nets de l'Énéide par exemple, que l'on peut placer entre toutes les mains à différents moments de l'apprentissage avec un minimum de préparation. Cette difficulté, visible dès la lecture ovidienne du Moyen Âge, ne s'est pas résolue au fil du temps : les cursus scolaires de la Renaissance et de l'âge classique l'ont connue également, cherchant des biais pour lire un auteur dont les collèges ont toujours usé de manière à la fois assidue et embarrassée.

Cet embarras relève à la fois de la pratique didactique et de positions idéologiques sur la finalité de la lecture des textes anciens. Plusieurs facteurs structurels poussent à une lecture rarement suivie de l'œuvre d'Ovide. De nombreux textes favorisent une lecture parcellaire ou sous la forme du florilège : les Hérö̈des, par exemple, sont composées d'élégies formant chacune un tout, et quelle que soit la réelle volonté de construire le recueil de la part du poète, il est facile de le déconstruire et de se limiter à la lecture de quelques pièces, chacune des lettres formant le recueil pouvant être comprise indépendamment des autres. Par ailleurs, les Métamorphoses forment un bloc dont précisément la longueur massive rend difficile la lecture suivie de la macro-structure, tandis que la microstructure des récits enchâssés ou liés permet une lecture par fragments. 
Enfin, des textes comme l'Art d'aimer, jugés licencieux ou sulfureux, ont paru impossibles à offrir tels quels en classe à des enfants ou des adolescents : l'attitude du maître a été soit de les écarter simplement du corpus scolaire, soit de les intégrer en les démembrant ou en les recomposant, ce qui en permet le contrôle mais leur ôte toute cohérence d'œuvre homogène.

Nous observerons cette attitude de la Renaissance jusqu'au début du $\mathrm{XVIII}^{\mathrm{e}}$ siècle, mais elle n'est pas propre à cette période: les lettrés des siècles précédents ne lisaient pas Ovide en entier, pour des raisons autant matérielles (toutes les œuvres n'étaient pas accessibles) que morales. Le poète est connu en latin et dans les langues romanes, mais dans une lecture philosophique qui voit dans ses textes une préfiguration de la vérité chrétienne, voie facilitée par des scènes comme celle du déluge inaugural des Métamorphoses. D'autres textes font l'objet d'une lecture sociale et morale qui, dans l'Ars Amatoria ou dans les Héroïdes, cherche l'exaltation d'un amour marital et chaste comme celui de Pénélope, ou la condamnation de l'amour passionnel et fou de tant d'autres héroïnes. Le texte des Métamorphoses, de son côté, est découpé en unités mythologiques, qui sont allégorisées comme autant de représentations de l'âme chrétienne et de la vérité divine ${ }^{1}$.

La Renaissance et l'âge classique héritent de cette lecture fragmentée et gauchie, et ne s'en départiront pas dans l'approche scolaire du poète, même si la culture humaniste docte donne à Ovide une place de choix. À partir des débuts de l'imprimé, on voit apparaître deux sortes d'éditions : des éditions savantes et érudites, dont celles des œuvres complètes dès $1492^{2}$, et des éditions d'extraits

1 Sur cette question, voir les nombreux travaux de Marylène Possamaï, dont, pour une présentation générale, Ovide au Moyen Age, 2008, dans la base HAL (halshs-00379427, consultable sur https:/halshs.archives-ouvertes.fr/halshs-00379427) et plus précisément, M. Possamaï-Perez, “ Comment raconter et interpréter au Moyen Âge les récits d'agression sexuelle de la mythologie antique? », in C. Bonnet, C. Noacco, J.-P. Aygon (éds), La mythologie de l'Antiquité à la modernité: Appropriation-AdaptationDétournement [URL : http://books.openedition.org/pur/39606, consulté pour la dernière fois le 31 juillet 2018], Rennes, 2009, p. 183-196 ; ainsi que M. Possamaï, M. Besseyre (éds), L’Ovide Moralisé illustré, Cahiers de Recherches Médiévales et Humanistes 30 (2015), p. 13-219, ainsi que, dans ce numéro, l'article de B. Nelis, “ D’un Ovide chrétien à un Ovide burlesque, du Moyen Âge au Grand Siècle ", notamment à propos de la lecture chrétienne de l'épisode de Pyrame et Thisbé.

2 Les premières éditions d'Ovide datent de 1490 (éditions des élégies d'avant l'exil, notamment De remedioamoris et Hérö̈des ; voir USTC 747644, 761517, 992676, 992708, 992726). L'édition de 1492 paraît à Venise chez Lazarus de Suardis de Saviliano, en deux volumes, comprenant pour le premier les Métamorphoses éditées par Bono Accorsio, et pour le second : Amores ; Ars amandi; De pulice ; Remediaamoris ; Heroides ; De nuce ; Medicaminafaciei ; Ibis ; Tristia ; Sappho adPhaonem ; Epistolae ex Ponto ; Fasti ; et du Pseudo-Ovide la Consolatio ad Liviam (USTC 992712). 
très « retravaillés », destinées au milieu scolaire, qui seront l'objet de mon étude. Avant d'en décrire les principales impressions, je présenterai la place donnée à Ovide dans les cursus scolaires, que nous pouvons connaître par quelques textes programmatiques réglant les études des collèges ou académies du Xvi ${ }^{\mathrm{e}}$ siècle au début du XviII ${ }^{\mathrm{e}}$ siècle.

\section{De Sturm à Rollin : un auteur inévitable mais embarrassant}

Les rationes docendi que nous avons conservées apparaissent quand l'enseignement collectif s'organise officiellement dans l'aire réformée comme une marque d'identité et d'indépendance de cités qui n'ont pas d'université et ne veulent pas laisser à d'autres le soin d'éduquer leurs citoyens ${ }^{3}$. Mais ces programmes, comme souvent les textes théoriques, organisent les études à partir d'un réel déjà partiellement existant, qu'ils reflètent en même temps qu'ils l'amendent ou le fixent. Les textes qui paraissent à partir de 1538 et jusqu'à la fin du siècle, quand les collèges jésuites répondent à la concurrence des Académies, sont donc aussi appuyés sur la pratique des écoles du début du siècle, particulièrement pour les premières années de la formation, celles du collège ou de la schola priuata, avant les années que nous appellerions universitaires, de la schola publica. L'école des Frères de la Vie Commune à Deventer, autant que les collèges français, parisiens ou non, fournissent des modèles à imiter ou à infléchir; souvent aussi, des enseignants qui ont exercé dans ces établissements passent dans les nouvelles Académies, notamment des Français sympathisants de la Réforme qui quittent le royaume de François I ${ }^{\text {er }}$ ou Henri II pour la Suisse ou le bassin alémanique. C'est le cas par exemple de Mathurin Cordier, d'abord enseignant dans plusieurs collèges parisiens (dont le collège de la Marche et le collège de Navarre), puis au collège de Guyenne à Bordeaux, avant d'arriver à Genève en 1536 et d'être l'un des inspirateurs et probablement rédacteurs des règles de l'Académie de Lausanne ${ }^{4}$.

3 Venant après les Académies de Strasbourg et Lausanne, les Leges Academiae Geneuensis, promulguées en 1559, insistent particulièrement sur ce point dans la version latine et intégrale du texte : ... ad eum ssque diem coacta fuisset ciuitas Geneuensis, [...] ab iis vrbibus \& gentibus petere suae iuuentuti bonarum artium ac disciplinarum cognitionem, quibus ipsa quod longe maximum est, id est syncerae religioniis scientiam, [...] largiebatur. Visa est Dei bonitas ac misericordia eo die concedere huic ciuitati quod paucissimis antea contigit, vt una eademque vrbs \& pietatis \& eruditionis parens haberi posset. («Jusqu'à aujourd'hui la cité de Genève [...] a été contrainte de demander pour sa jeunesse la connaissance des bonnes lettres à des cités et des peuples auxquels elle offrait [...] ce qui est de loin le plus important, c'est à dire la science de la vraie religion ; il a paru bon à la bonté et à la miséricorde de Dieu de concéder aujourd'hui à cette cité ce qui est arrivé auparavant à très peu, c'est à dire qu'une seule et même ville puisse être tenue pour leur mère en piété et en érudition. »)

4 Sur Cordier, voir J. Le Coultre, Mathurin Cordier et les origines de la pédagogie 
Le premier texte qui détaille un programme d'études pour les jeunes gens depuis l'enfance jusqu'à l'entrée dans l'âge adulte est le De literarum ludis recte aperiendis liber de Jean Sturm, paru en 1538, qui donne le cadre formel des études au Gymnase de Strasbourg. Ce texte, à ce moment ou lorsqu'il sera repris par son auteur en 1558, place une première lecture des poètes pendant la septième classe, soit la troisième année des études du collège, au moment où les enfants, qui ont alors environ 10 ou 11 ans, maîtrisent suffisamment les structures de la langue latine pour qu'on les initie à la poésie ${ }^{5}$. Ovide n’est pas mentionné, mais Catulle, Tibulle et Horace le sont après Virgile, les deux premiers cependant avec la restriction qu'ils doivent être « pudiques », car « rien qui ne soit pudique, pieux, élégant et noble, ne doit entrer dans les oreilles et l'esprit d'un enfant „ .

Ovide apparaît dans le programme des étudiants avancés de la schola publica, mais comme un auteur presque toujours facile, qui peut et doit donc être lu chez soi : mention particulière est faite des Fastes, à la fois utiles, probablement pour

protestante dans les pays de langue française : 1530-1564, Neuchâtel, 1926 ; K. Crousaz, L'Académie de Lausanne entre humanisme et Réforme (ca. 1537-1560), Leiden/Boston, 2012, p. 416-436 notamment ; M. Furno, "Quod aliquando fuit, potest instaurari : parler latin au xvI ${ }^{\mathrm{e}}$ siècle, une restitution en trompe-l'œil? ”, Anabases 17 (2013), p. 105118 , et « Du cours volé au testament pédagogique: Mathurin Cordier et le choix de l'imprimé », in G. Houtz (éd.), Nouveaux aspects de la culture de l'imprimé. Questions et perspectives, $X V^{e}-X V I I^{e}$ siècles, Genève, 2014, p. 344-361.

5 Il n'est en rien étonnant que deux années aient suffi aux enfants pour apprendre à lire et écrire, et savoir les rudiments du latin. Même si nous n'avons pas de repères précis pour évaluer la réussite exacte d'une telle pédagogie, le texte de Sturm comme celui des maîtres de Genève nous montre que les enfants à ce stade étaient adonnés à cette étude au moins quatre heures par jour, six jours sur sept et onze mois sur douze. Il était donc plus facile d'apprendre rapidement l'essentiel de la langue latine qu'au cours des 48 heures académiques dévolues annuellement, aujourd'hui, à un cours de latin dans la plupart des universités françaises.

6 Il existe de ce texte une édition moderne avec traduction française, à laquelle je n'ai pas eu accès: J. Sturm, De literarum ludis recte aperiendis liber. De la bonne manière d'ouvrir des écoles de lettres, traduction de G. Lagarrigue, postface de M. Arnold, Strasbourg, 2007. Mes références sont données sur l'édition de Strasbourg, Wendelin Rihel, 1538, la traduction me revient ; fo $15 \mathrm{r}-\mathrm{v}$ : Aeneis Virgiliana uirtutes omnes heroici uersus, reliqua genera ex Catullo et Tibullo et Horatio sunt congerenda. Plura ex Catullo et Tibullo si essent pudica... inuenias. Sed nihil in pueri aures aut animum intret quod non sit pudicum, pium, elegans, liberale. (“ L'Énéide de Virgile contient toutes les vertus pour le vers héroïque ; les autres genres doivent être rassemblés dans Catulle, Tibulle et Horace. Tu trouverais plus chez Catulle et Tibulle s'ils étaient pudiques. Mais rien qui ne soit pudique, pieux, élégant et noble, ne doit entrer dans les oreilles et l'esprit d'un enfant. ») 
leur contenu documentaire et historique, et difficiles, et des Métamorphoses, indispensables à la culture, mais qui “ encombrent» le cursus académique. On voit bien ici l'attitude ambivalente du pédagogue devant un auteur qu'on ne peut ignorer, qui « doit tout à fait être lu » ${ }^{7}$, mais qu'on renvoie à une étude privée, dont le maitre ne prend pas la responsabilité, parce que ses textes, lus en continu, n'entrent pas dans «ce qui est pudique, pieux, élégant et noble». Sturm luimême confirmera cette pratique : bien après le texte programmatique, il donne un manuel de poésie, qui se présente comme six livres de textes de divers auteurs, compilés selon leur niveau de difficulté mais également en fonction de l'âge des enfants auxquels ils sont destinés, et de la morale qu'on veut leur inculquer. Le premier livre s'adresse à la sixième classe, ou quatrième année, du collège de Strasbourg, dont le maître est Martin Malleolus : Ovide n'est pas absent de la compilation, mais il y est rare, et toujours significativement amputé. Par exemple, on trouve pour ce niveau la citation d'Ovide, artibus ingenuis quae sita est gloria multis (" beaucoup ont cherché la gloire dans les arts libéraux»), mais sans le second vers du distique, infelix perii dotibus ipse meis (« moi-même, malheureux, j'ai péri par mes propres dons »), évocation par le poète de ses vers élégiaques qui lui auraient coûté l'exil ${ }^{8}$. Nous voyons ici l'application pratique de ce qui n'est que formulé de manière générale dans le texte du De literarum ludis recte aperiendis liber : Ovide est indispensable à la culture humaniste, mais il doit être lu par des adultes responsables, qui devront filtrer cette lecture pour de jeunes écoliers.

Quelques années plus tard, l'Académie de Lausanne en 1547, puis celle de Genève en 1559, se dotent de Leges Academiae, qui sont très proches l'une de l'autre à propos d'Ovide. À Lausanne, les textes du poète sont introduits dans la troisième classe, soit la cinquième année d'étude, quand les enfants ont 11 ou 12 ans environ. Les textes proposés sont les Tristes et les Pontiques, à étudier en alternance avec l'Énéide de Virgile. À Genève, ce sont aussi les Tristes et les Pontiques qui sont utilisés, mais un an plus tôt, en quatrième classe, et comme support explicite pour l'apprentissage des “quantités des syllabes, réduites à un petit nombre de règles ", sans doute la raison également de leur mise au programme à Lausanne: ces textes permettent de travailler la métrique du distique sans toucher à des élégies amoureuses, et peuvent donc également être

7 Sturm, De literarum ludis, 1538, fo 26v-27r : Ouidius prope in omnibus facilis est, ideoque relinquendus bibliothecis et legendus domi. Fastorum tamen libri et utilitatem habent, et difficultatem, Metamorphosis legi omnino debet, at scholae curriculum impedit. " Ovide est un auteur presque toujours facile, et pour cette raison doit rester en bibliothèque et être lu chez soi. Les Fastes cependant présentent une utilité, et de la difficulté. Les Métamorphoses doivent tout à fait être lues, mais encombrent le cursus scolaire. »)

8 Ovide, Pontiques, II, 7, 47-48; dans Poetica sex volumina, cum lemmatibus Johanni Sturmii in usum scholarum Argentinensium, Strasbourg, Josias Rihel, 1565, vol. I, p. 9. 
lus en intégralité, sans fragmentation qui évite au jeune lecteur tout contact avec un passage « dangereux ». Aucune mention n'est faite d'une autre œuvre du poète, ni dans le reste des programmes de la schola priuata ni dans ceux de la schola publica. Là encore, l'étudiant qui connaît d'autres élégies ou Métamorphoses ne peut les avoir rencontrées que lors de ses propres lectures, et non en classe : les maîtres laissent à la maison, ou aux bibliothèques, le soin de nourrir cette partie de la culture littéraire des élèves ${ }^{9}$.

Enfin, quand les Jésuites mettent en forme également, par plusieurs versions successives, la Ratio studiorum des collèges de l'ordre, la place d'Ovide est à peu près la même. Le poète est préconisé pour la classe moyenne de grammaire, qui correspond sensiblement à un public de même âge et de même niveau que celui des quatrième ou troisième classes des Académies réformées ; il partage le temps de lecture avec les Familiares de Cicéron exclusivement, et seuls les « poèmes les plus faciles » sont proposés ${ }^{10}$. Nous retrouvons donc une lecture d'apprentissage, mais ici uniquement par fragments, laissant au soin des maîtres de sélectionner les textes qu'ils lisent en classe, tant pour la difficulté de la langue que pour la moralité. Cette précision est implicite dans la Ratio jésuite, sans doute parce que ce mode de travail est évident pour les enseignants. Il ne variera pas tout au long du XvII $^{\mathrm{e}}$ siècle, la Ratio ne subissant pas de changements profonds. Quelle que soit donc leur confession, les pédagogues des $\mathrm{XVI}^{\mathrm{e}}$ et $\mathrm{XVII}^{\mathrm{e}}$ siècles se retrouvent d'accord a minima autour d'un poète intéressant, mais difficile voire dangereux, à ne pratiquer qu'avec précaution : soit les enfants ne lisent en entier que certains titres sans portée sentimentale, soit leur lecture est plus large en apparence, mais elle ne concerne que des flores selecti.

Cette défiance envers Ovide ne semble qu'aller croissant avec l'installation de la Contre-Réforme, et d'une pensée de plus en plus rigoureuse, voire rigoriste, sur la morale éducative et la lecture des textes anciens. Au début du Xviı ${ }^{\mathrm{e}}$ siècle, les

9 Les Leges de l'Académie de Lausanne sont éditées et traduites dans le volume de Crousaz, L'Académie de Lausanne. Pour Ovide, voir p. 486-487 : Inter poetas Ouidius de Tristibus aut de Ponto, et Aeneis Virgilii vicissim proponuntur ( Parmi les poètes, que l'on commente tour à tour Ovide, les Tristes ou les Pontiques, et l'Enéide de Virgile »). Pour Genève, voir Leges Academiae Genevensis, Genève, Robert Estienne, 1559, $\mathrm{f}^{\circ} \mathrm{c} 1 \mathrm{v}$ : Explicentur quoque syllabarum quantitates, paucis regulis comprehensae cum Ouidii Elegiis de Tristibus et de Ponto (“Qu’on explique aussi les quantités des syllabes, réduites à un petit nombre de règles, avec les Tristes d'Ovide ou les Pontiques »).

10 Voir Ratio studiorum. Plan raisonné et institution des études dans la compagnie de Jésus, présentée par A. Demoustier et D. Julia, traduite par L. Albrieux et D. Pralon-Julia, annotée et commentée par M.-M. Compène, Paris, 1997, p. 185 : Ad praelectiones vero non nisi familiares Ciceronis epistolae, et facillima quaeque Ouidii carmina (« Pour les prélections, exclusivement les Lettres familières de Cicéron et les poèmes les plus faciles d'Ovide »). 
liminaires d'une des éditions scolaires nous renseignent clairement sur le débat qui divise les pédagogues, certains permettant une lecture plus large d'Ovide, au titre de la culture générale, d'autres l'excluant complètement des classes. La page de titre du recueil Locutionum et sentientiarum Flores [... e e Latinorum principibus Virgilio, Horatio et Ovidio collecti, paru à Halle en 1702, précise bien que « la préface enseigne pour quelles raisons les écrits des Païens doivent et peuvent être lus dans les écoles chrétiennes ", mais cette préface s'attarde essentiellement (douze pages sur quatorze) à montrer, avec une rhétorique véhémente, les dangers qu'il y a à faire lire en entier, sans discernement, les poètes païens aux enfants chrétiens. Les deux dernières pages justifient comme seule possible la lecture démembrée, par Flores, qui protège du danger puisqu'un maître responsable a rigoureusement sélectionné les extraits " ${ }^{11}$ Cette grande méfiance vis à vis d'Ovide se retrouve dans le Traitédes études de Charles Rollin, paru à Paris de 1726 à 1728 : dans son cursus, ce pédagogue janséniste accepte une lecture sélective des poètes, où les choix émanent de " personnes d'un âge mûr, d'une expérience consommée et d'une probité reconnue ${ }^{12}$. À cette condition, Ovide est acceptable pour apprendre à écrire à des débutants en métrique, car « ce poète est fort propre à inspirer du goût pour la poésie; à donner de la facilité, de l'invention, de l'abondance. Ses Métamorphoses surtout peuvent être fort agréables par la grande variété qui y règne », mais il reste que Virgile " fait la plus grande occupation des classes : aussi est-ce un modèle parfait et qui peut suffire à former le goût » ${ }^{13}$. Encore une fois, Ovide devient un manuel de métrique, ici hexamétrique puisqu'il s'agit des

1 LOCUTIONUM AC SENTENTIARUM || LATINARUM || FLORES, || Qui magnas Latinitatis opes || continent: || E Latinorum Poëtarum Principibus, || VIRGILIO, HORATIO || \& OVIDIO \| Collecti, \| Ac in gratiam juventutis Scholasticae, ad pa|randam tam solutce, quam ligatce Orationis || copiam ac facultatem, || SECUNDUM LIBRORUM SERIEM, || iterum editi,|| opera || JOACHIMI LANGII. || PRAEFATIO || Docet qua ratione Paganorum scripta in || Christianorum scholis legi possint ac || debeant. || CUM PRIVILEGIO || HALAE SAXONUM, || in officina ORPHANOTROPHII Libraria, || Berolini, Typis SCHLECHTIGERIANIS, 1702. Malgré la mention iterum editi, je n'ai pas retrouvé trace d'une édition antérieure à 1702, du moins avec ce titre. La préface occupe les $\mathrm{f}^{\mathrm{o}} * 2 \mathrm{r}$-[8v]. Ovide dans cette édition prend la même place que Virgile, soit soixante-quatre pages d'extraits : quarante-cinq pages offrent des citations plus ou moins longues tirées des Métamorphoses, des Pontiques, des Tristes, des Fastes et des Héroïdes, mais dix-neuf pages ensuite donnent des extraits non référencés des autres œuvres d'Ovide, dont l'Art d'aimer, sans qu'il soit possible de lire le texte avec son sens d'origine.

12 C. Roluin, Traité des études. De la manière d'enseigner et d'étudier les Belles Lettres par rapport à l'esprit et au coeur, Paris, Le Normand, 1807, p. 150.

13 RoLuin, Traité des études, p. 170. 
Métamophoses : ce choix pédagogique est sans doute motivé par le fait que le texte se prête bien à un découpage en extraits nombreux sur des sujets fabuleux, mieux capables de captiver les plus jeunes élèves que le grandiose épique virgilien. Pas plus que deux siècles auparavant, on ne lit Ovide en entier, pour toutes ses œuvres et de manière suivie.

\section{De Hermann Torrentinus à Daniel Crespin : une impossible lecture suivie}

Les manuels qui utilisent Ovide pour l'apprentissage du latin dans les classes présentent, comme les textes programmatiques, une grande unité, quel que soit le maître qui les compose : il s'agit de ne pas se priver du support pédagogique que peuvent fournir les poèmes ovidiens, mais d'en déjouer les dangers par une lecture filtrée - au point parfois de dénaturer le texte d'origine.

Un des premiers manuels qui met Ovide à l'épreuve de la classe sont les Orationes familiares et elegantissime ex omnibus Publii Ouidii libris formatae, mises en forme par Hermann Van der Beeke ou Torrentinus. Ce pédagogue entré chez les Frères de la Vie Commune à Deventer enseigne dans leurs écoles à Groningue et à Zwolle, et rédige pour ses classes de petits manuels de grammaire, des éditions avec gloses de Virgile et des hymnes à la Vierge Marie de Sabellicus, et l'Elucidarius poeticus, dictionnaire de noms propres mythologiques et de mots grecs, qui sera constamment repris, augmenté et réimprimé par divers auteurs dans le courant du xvI ${ }^{\mathrm{e}}$ siècle ${ }^{14}$.

Les Orationes familiares sont un bon livre de classe dans une petite aire germanique au début du xvi ${ }^{\text {e }}$ siècle : publié d'abord à Zwolle par Arnold Kempen en 1502, le livre est repris à Cologne en 1505 chez Martin von Werden, puis chez le même imprimeur en 1509, 1510, en 1510 encore chez Heinrich Quentel à Cologne, en 1513 chez les deux précédents de nouveau, en 1516 et 1519 à Leipzig chez Valentin Schumann, et en 1521 de nouveau à Cologne chez Quentel ${ }^{15}$. Le manuel aura donc duré une vingtaine d'années, avant d'être abandonné, non qu'il ait été remplacé par un autre mais parce qu'il ne correspondait plus aux programmes des petites classes de débutants auxquelles il était destiné.

14 Voir P. Bietenholz (éd.), Contemporaries of Erasmus, Toronto, 2003, vol. 3, p. 331-332. Les editiones principes de ces ouvrages paraissent à Deventer entre 1492 et 1498.

15 Pour la princeps, voir USTC 420050. La dernière parution isolée semble être en 1538 à Anvers, chez Michaël Hoochstraten. Sur un passage du manuel, voir P. Maréchaux, "La réception de Protée dans les mythographies et les commentaires d'Ovide entre 1350 et 1550 : l'histoire d'une dissociation ", in A. Rolet (éd.), Protée en trompe l'oeil: Genèse et survivances d'un mythe, d'Homère à Bouchardon, Rennes, 2010, p. 347-364. 
En effet, le manuel utilise Ovide hors de la connaissance de la métrique, ce qui le destine clairement aux débuts de l'apprentissage du latin, probablement aux deux premières années. Le manuel se présente comme une suite de sentences moralisantes, en prose des plus plates, mettant en scène des personnages d'enfants dans lesquels les jeunes élèves pourront se projeter (Ioannes, qui n'est pas toujours exemplaire, ou Paulus, Petrus et Andreas, plus sages). En emboîtant les phrases les unes aux autres, l'auteur obtient des micro-récits supposés aider à la mémorisation, dans une expression conçue pour la modélisation de l'écriture. Les sentences avancent donc en blocs thématiques de quelques lignes par associations d'idées, et permettent d'apprendre la variation stylistique ou un début de copia verborum, comme dans l'exemple suivant :

Ioannes inuitis parentibus et cognatis Romam profectus est.

“ Jean est parti à Rome contre la volonté de ses parents et de ses proches. »

Equidem inuitis omnibus hoc faciam.

“Assurément, je ferai cela contre la volonté de tous. »

Velis nolis id facies.

“ Tu le feras que tu le veuilles ou non. »

Velimus nolimus moriendum nobis est.

“Que nous le voulions ou non, il nous faut mourir. »16

Les blocs de sentences sont coupés entre eux par des gloses de Torrentinus qui éclairent certains termes jugés difficiles, et l'étayage se fait parfois par une traduction en bas-allemand, ce qui confirme l'utilisation du livre dans les petites classes. Par exemple, la sentence Sum defessus stando sed indefessus audiendo est glosée par defessus, moede, indefessus, niet moede ${ }^{17}$. Mais la glose peut être uniquement latine à d'autres endroits, comme lorsque Arida ramalia est expliqué par Ramale est ramus arboris ${ }^{18}$. Visuellement, il est parfois difficile de retrouver la glose correspondant à un vers, la typographie des premières éditions étant compacte, et la raison qui amène une glose sur un mot plutôt que sur un autre n'est pas toujours clairement identifiable, si ce n'est par l'usage et la pratique de ces phrases devant des enfants.

16 H. Torrentinus, Orationes familiares, Köln, Martin von Werden, 1505, fo A1v.

17 Torrentinus, Orationes familiares, $\mathrm{f}^{\circ} \mathrm{C} 4 \mathrm{v}$.

18 Torrentinus, Orationes familiares, $\mathrm{f}^{\mathrm{o}} \mathrm{C} 2 \mathrm{r}$. 
À première vue, Ovide semble bien absent de toutes ces phrases, tant la prosification et la décontextualisation éloignent le texte de référence. Pourtant, le titre du manuel n'est pas un mensonge, et Torrentinus avait une bonne connaissance du poète : pour reprendre les exemples ci-dessus, l'adjectif inuitus est employé par Ovide de très nombreuses fois, et la locution inuitis omnibus apparaît au vers 107 de la première Héroïde, dans un contexte qui rappelle l'idée d'un départ exprimée dans la sententia précédente ${ }^{19}$; de même, ramalia arida apparaît dans l'épisode de Philémon et Baucis des Métamorphoses ${ }^{20}$, et la phrase forgée par Torrentinus, Arida ramalia bene fouent ignem ( $₫$ Les rameaux secs alimentent bien le feu »), est une glose exacte du passage d'origine.

Comme le dit également la page de titre, au-delà de ces phrases familières forgées à partir d'expressions ovidiennes, on trouve aussi par endroits des versus quidam integri notatu digni (« quelques vers entiers dignes d'être notés »). Ils sont assez facilement repérables, le rythme métrique tranchant sur la prose des autres sententiae.

Par exemple, au fo A2v, on trouve le texte suivant :

Heu patior telis vulnera facta meis.

Exitus acta probant.

Non sunt ab euentu estimanda facta.

Perniciosum est blandis credere verbis.

“ Hélas, je souffre des blessures faites par mes propres traits.

La fin justifie les actes.

Les faits ne doivent pas être appréciés en fonction de leur réalisation.

Il est pernicieux de croire aux douces paroles. »

qui est un montage à partir de deux passages de la lettre de Phyllis des Héroides, pour partie cités exactement et pour partie résumés, soit Héroïdes, II, 45-46 (je souligne) :

Heu! Patior telis vulnera facta meis!

Credidimus blandis, quorum tibi copia, verbis.

“ Hélas, je souffre des blessures faites par mes propres traits! Nous avons cru en tes douces paroles, dont tu as abondance. »

19 Ovide, Héroïdes, I, 107 : dum parat inuitis omnibus ire Pylon (« tandis qu'il [Télémaque] se préparait à partir pour Pylon à l'insu de tous »).

20 Ovide, Métamorphoses, VIII, 644-645: multifidasque faces aridaque ramalia tecto / detulit. Le passage met en scène Baucis allumant un feu. 
et Hérö̈des, II, 83-85 :

Atque aliquis 'iam nunc doctas eat' inquit 'Athenas :

armiferam Thracen qui regat, alter erit.

Exitus acta probat.' [...]

“Et un autre lui dit: "Va donc désormais jusqu'à la savante Athènes: un autre gouvernera la Thrace porteuse d'armes. La fin justifie les actes.” »

Le poète fait dire ici à son héroïne qu'elle pâtit de ses actes, mais les orationes disent légèrement autre chose, plus sentencieusement, à savoir qu'il faut se méfier des beaux parleurs pour ne pas risquer d'être puni de sa crédulité. Le tragique qui faisait la grandeur du texte poétique est réduit à une recommandation grondeuse et un peu plate pour de jeunes imprudents. Le texte fourmille de récritures de passages amoureux, de quelque objet qu'ils parlent, qui tentent de détourner les yeux des jeunes lecteurs de ces textes dangereux. Les Orationes poussent en fait à l'extrême les deux caractéristiques de la lecture médiévale d'Ovide, c'est à dire la moralisation et la lecture fragmentée, indissociables l'une de l'autre.

Le manuel de Torrentinus cesse d'être fréquemment utilisé après 1530 , probablement parce que les maîtres renoncent à utiliser Ovide dans les premières années d'apprentissage. Presque en même temps et au même endroit, Johannes Murmellius ${ }^{21}$, autre pédagogue formé à Deventer et ami de Torrentinus, fournit un recueil d'extraits de Tibulle, Properce et Ovide : la première édition paraît à Deventer en 1504, et est très sommaire ${ }^{22}$. Elle réunit, sous des tituli qui sont autant de loci communes, 45 pages in quarto de distiques extraits des trois poètes, mêlés sans distinction ni d'œuvre ni d'auteur, mais gardés dans leur forme métrique. Le livre s'ouvre sur un petit texte en distiques de Murmellius lui-même, qui donne clairement le ton de l'ouvrage : il s'agit de permettre que les enfants lisent ces auteurs, mais sans les dangers que les passages érotiques ou amoureux peuvent faire courir à leurs esprits ${ }^{23}$. Le fait que les textes soient conservés dans leur forme métrique destine l'ouvrage aux classes un peu plus avancées où les enfants commencent à apprendre à écrire de la poésie, et l'ouvrage de Murmellius, plus adapté à une lecture humaniste qui respecte la forme des auteurs anciens, va avoir un très grand succès dans toute l'aire réformée jusqu'à la fin du siècle. Le titre changera de forme selon les imprimeurs, et deux lignées d'éditions verront le jour, l'une avec le poème en distiques de Murmellius, l'autre, à partir de 1513, avec une

21 Voir Bietenholz, Contemporaries of Erasmus, vol. 2, p. 470-471.

22 Ex elegiis Tibulli Propertii et Ouidii ab Joanne Murmellio selecti uersus, [Deventer, Jacob de Breda,] 1504.

23 Ex elegiis [...] selecti uersus, 1504, $\mathrm{f}^{\circ} \mathrm{Ai} \mathrm{v}$ : Hos puer euites moneo ne blanda uoluptas / te miserum cogat turpia ferre iuga ( Je te préviens, enfant, d'éviter ces poètes, pour que la douce volupté ne te contraigne pas, malheureux, à porter un joug honteux »). 
dédicace de Murmellius à Theodoricus Tzwyvel, qui éclaire encore le contexte de rédaction du manuel : Tzwyvel avait réclamé une anthologie d'Ovide, à laquelle Murmellius a ajouté les deux autres auteurs, dont les œuvres, parce qu' « elles sont pleines de mollesse, de lascivité et d'amour », “ ne doivent pas être expliquées [ou : lues en entier] à l'âge tendre „24. Le florilège permet donc d'en accepter la lecture, accompagnée d'argumenta qui la facilitent. De plus, Murmellius dit s'être tenu au respect des ouvres, refusant de confondre les textes et de les altérer, bien qu'il ait conscience que l'anthologie puisse quelquefois modifier le sens du texte ${ }^{25}$. Les éditions postérieures à 1530 enrichiront la matière, la rangeront en faisant apparaître le nom des auteurs et l'ordre de leurs œuvres, et le livre, sous le titre Flores Tibulli, Propertii ac Ouidii, ou sous celui Loci communes sententiosorum versuum ex elegiis Tibulli, Propertii ac Ouidii, sera réimprimé et utilisé jusqu'au $\mathrm{XVII}^{\mathrm{e}}$ siècle. Murmellius a donc pérennisé au moins les principes de Torrentinus : la lecture des extraits est orientée par les argumenta, et on y retrouve souvent les mêmes vers glosés de la même façon. Par exemple, sous l'argumentum Facta a causis, non ab euentu sunt uel probanda uel improbanda (« Les faits doivent être approuvés ou désapprouvés par leurs causes, non par leur réalisation »), on retrouve le distique des Hérö̈des, II, 85-86, Exitus acta probat, careat successibus opto / quisquis ab euentu facta notanda putat ( La fin justifie les actes, je souhaite qu'il soit privé de succès, celui qui pense que les faits doivent être pesés à partir de leur réalisation »), qui pousse à se méfier des deceptores puellarum évoqués dans l'argumentum précédent.

Le principe une fois établi, la concurrence se multiplie, et apparaissent selon le même modèle des ouvrages consacrés au seul Ovide, avec des titres proches, Sylva sententiarum ex Ovidio, ou Flores seu sententiae morales ex Publii Ovidii Nasonis operibus, dont on ne sait pas toujours qui est le compilateur. Ces textes

24 Varia Tibulli Propertii et Ouidii carmina, T. Maler, Erfurt, 1513, titre verso : [Opera] sic molliciei, lasciuiae, ac Veneris referta, ideoque vernanti aetati minime praelegenda. Certaines éditions comme celle de Gymnicus, Köln, 1537, portent perlegenda et non praelegenda, deux sens possibles ici, la pratique scolaire ne pouvant ni lire en entier ni expliquer ces textes.

25 Varii Tibulli Propertii et Ouidii Flores, titre verso; Neque tamen (quod quidam audaculi fecerunt) poetarum carmina confudi, aut immutaui, sed eo ordine eaque integritate qua apud ipsos autores legitur reposui. Quod si pauca in alienum sensum deflexerim, nemini mirum uideatur, cum id nec ab re factum sit, nec auctoritate careat. ( Je n'ai cependant pas mélangé, ou modifié, les vers des poètes - ce que quelques petits audacieux ont fait - mais je les ai rapportés dans l'ordre et dans l'intégrité où on les lit chez ces poètes. Si quelquefois je les ai tournés dans un sens différent, que cela n'étonne personne, puisque ce n'a pas été fait sans raison, et ne manque pas d'être autorisé [par l'exemple de St Jérôme]. ») 
fonctionnent tous de la même façon, par extraits qui évitent tous les passages délicats et orientent vers une lecture moralisée du texte. Leurs différences tiennent dans la technique de compilation : les Flores arrangées par Andreas Diether en $1548^{26}$ sont au sens strict une anthologie, c'est-à-dire que, même précédés d'un argumentum moral, les extraits sont rapportés en l'état, en suivant leur ordre dans le texte d'origine, et la succession des recueils dont ils sont tirés est toujours la même, même si pour chaque sujet tous les recueils ne sont pas sollicités : Tristes et Pontiques, puis Fastes, Hérö̈des et Ars amatoria, les Tristes et les Pontiques, dont nous avons vu qu'ils sont mis au programme par les Académies de Lausanne et Genève, étant les plus utilisés.

Mais la Sylva sententiarum ex Ovidio, dont le compilateur anonyme est peutêtre visé par la préface-dédicace de Murmellius, compose de son côté un centon redoutable, parfois peu utilisable même comme modèle métrique tant les œuvres sont confondues. Par exemple, sous l'argument De patientia, on trouve le texte suivant ${ }^{27}$ :

\begin{tabular}{|c|c|}
\hline Posse pati facile est tibi ni patientia desit. & $=$ Ovide, Remèdes à l'a \\
\hline $\begin{array}{l}\text { Est aliquid magnis crimen abesse malis. } \\
\text { iter ex merito quicquid patiare ferendum est }\end{array}$ & $=$ Ovide, Fastes, I, 483 \\
\hline Quae venit indigne poena dolenda venit. & $=$ Ovide, Héroüdes, V, 7-8 \\
\hline $\begin{array}{l}\text { Equo animo poenam qui meruere ferunt. } \\
\text { mala Nasonem quoniam meruisse videtur }\end{array}$ & $=$ Ovide, Amours, II, 7, 12 \\
\hline si non ferre doles at meruisse dol & $=$ Ovide, Pontiques, I, 7, 67-68 \\
\hline
\end{tabular}

«Il est facile de pouvoir supporter si la patience ne te manque pas.

C'est quelque chose que de s'éloigner du crime dans de si grands malheurs.

Il faut supporter avec légèreté tout ce qu'on subit de façon méritée ; mais le châtiment qui vient de manière indigne est une douleur.

Ceux qui l'ont mérité supportent leur châtiment d'une âme égale.

et puisqu'il semble que Nason a mérité ses malheurs, si tu n'es pas malheureux qu'il les supporte, au moins sois malheureux qu'il les ait mérités. »

Comme le montre le tableau ci-dessus, ce passage combine des vers isolés, qui produisent une forme métrique fausse (deux pentamètres successifs au milieu des distiques), et qui en arrivent à faire dire au poète lui-même qu'il mérite d'avoir

26 Flores seu sententiae morales ex Publii Ovidii Nasonis [...], Augsbourg, Philippe Ulhard, 1548, compilées par A. Diether, poète, philologue, et pédagogue, auteur notamment d'un Thesaurus contexendarum epistolarum ad Ciceronem imitandum.

27 Sylua sententiarum ex Ovidio non librorum sed rerum ac Titulorum ordine seruato delectarum, Leipzig, Jacob Thanner, 1515, $\mathrm{f}^{\mathrm{b}} \mathrm{C} 3 \mathrm{v}-[\mathrm{C} 4 \mathrm{r}]$. 
été puni. La trame ainsi composée dit qu'il est facile de supporter les difficultés lorsqu'on a de la patience, et qu'on sait s'abstenir du crime dans le malheur; on peut même supporter légèrement ce qu'on mérite, et la peine douloureuse est celle qui est indigne, mais celle qui est méritée se supporte fermement ; et cela est finalement le cas du poète lui-même, dont on torture le texte, et qui semble avoir mérité la dénaturation de son livre.

Au tournant du xvıI ${ }^{\text {e }}$ siècle, nous avons vu que la Ratio Studiorum des Jésuites ne baisse pas la garde devant Ovide. Comme le montre un Appendix à la version de 1591 de la Ratio, les Pères ne se départissent pas de l'attitude des autres pédagogues, de quelque confession qu'ils soient: Ovide et quelques autres “ auteurs peu honnêtes » doivent être lus, au titre de la culture générale, et pour éviter que leurs auditeurs « ne parai[ssent] tout à fait novices devant des matières dans lesquelles tous les autres élèves se vanteront d'être versés „ ${ }^{28}$. Pour cela, il faut expurger, et les Pères préconisent une forme d'édition qui va être leur « image de marque » et sera reprise jusqu'au xix ${ }^{\mathrm{e}}$ siècle pour divers auteurs. Plutôt que de compiler des extraits sous forme de centon, plutôt que de toucher le texte en en modifiant les termes, ce qui n'empêche pas « que le sujet indécent exhale toujours son odeur ou sa saveur primitive », il vaut mieux « supprimer les poèmes indécents », éventuellement en en réunissant les parties honnêtes, “ si, séparées $\mathrm{du}$ tout, elles peuvent garder leur cohérence » ${ }^{29}$. Ce texte donne le patron de tant d'éditions selectae et purgatae ad maiorem Dei gloriam, où le texte d'Ovide est certes moins travesti que dans les centons de la Sylua sententiarum, mais où il est toujours impossible de le lire dans son intégrité, et même d'imaginer celle-ci.

Par exemple, en 1595, l'édition des Héroïdes selectae et purgatae qui paraît chez Birckman à Cologne présente la lettre de Pénélope (Héroïde I) en entier, parce qu'elle continue de représenter le parangon de l'amour conjugal, mais la lettre d'Hermione (Hérö̈de VIII) est considérablement expurgée. Y sont supprimés les vers 37 à 40 qui sont une allusion à Ménélas, père indulgent aux amours de sa fille parce que lui-même adultère ; puis les vers 67 à 72, allusions aux adultères de Jupiter; enfin les vers 93 à 122, soit toute la fin du texte, où Hermione expose son déchirement entre deux amants. Mais ces coupures ne sont pas typographique-

28 Ratio Studiorum 1591, éditée dans L. Lukacs, Monumenta Paedagogica Studiorum societatis Iesu, t. 5, Roma, 1986, p. 332 : Genera [...] quae a parum honestis auctoribus tractata sunt; [...] quia auditores nostri novi prorsus vi debuntur ad ea, in quibus caeteri discipuli se iactabunt esse versatos. La traduction française de cet appendice est donnée dans Ratio Studiorum [...], Paris, 1997, p. 245-246.

29 Ratio Studiorum, Roma, 1986, p. 332 : semper tamen liber, cuius argumentum turpe sit, pristinum ac natiuum redolet odorem aut saporem [...] praetermissis turpibus poematis [...]. Ex poematis quorum argumenta obscoena sunt, possent $[. .$.$] colligi quaedam partes$ honestiores si a suo toto separatae cohaerere possent. 
ment apparentes : le texte se déroule sans marque de coupure, et sans connaissance de l'original, il est impossible de les repérer, car les distiques s'enchaînent sans chaos trop apparent. Seule la fin est abrupte et peut donner à penser.

Cette lecture morale et fragmentée perdurera longtemps : l'édition $A d$ usum Delphini donnée par Daniel Crespin, près d'un siècle plus tard en 1689, la véhicule toujours, quoique dans une portée moindre ${ }^{30}$. Ces éditions Ad usum Delphini ne sont pourtant pas conçues à l'usage des classes, mais pour le lectorat de l'honnête homme, adulte ou jeune homme, modelé par l'éducation idéale du Dauphin. Or, pour le premier volume qui dans cette édition contient les élégies amoureuses d'avant l'exil, le texte n'est pas lisible en continu : l'éditeur scientifique a renvoyé en fin de volume des obscoena qu'il signale dans le cours du texte par des étoiles (*****) et qui sont reproduits en fin de volume, référencés, et repositionnables dans le texte même. Il est donc possible pour un adulte, en 1689, de lire en entier ces textes d'Ovide, mais il n'est toujours pas possible de les lire en continu, puisque ce même texte reste fragmenté pour des raisons morales, et que la lecture complète suppose d'aller à la fin du livre et de revenir ensuite au texte dans son déroulement.

Les choses ont-elles radicalement changé de nos jours, en nos siècles « libérés »? Certes, les parcours universitaires de Lettres permettent de lire Ovide en continu, mais les catalogues des principaux éditeurs français depuis le début du xxi siècle ne comptent pas d'édition des œuvres complètes. Jean d'Ormesson est dans $L a$ Pléiade chez Gallimard, mais pas Ovide, et on trouve dans cette collection, pour les auteurs anciens, Homère, Virgile, divers historiens et philosophes, mais aucun des poètes que les Jésuites jugeaient "peu honnêtes ». Les éditions de textes d'Ovide, en latin ou en français, sont soit confinées chez les éditeurs spécialisés des littératures antiques, soit reprennent pour la plupart leurs traductions. Les catalogues offrent principalement des traductions des Métamorphoses, mais avec peu de choix si l'on souhaite une version complète, alors qu'abondent des versions d'extraits, notamment chez des éditeurs scolaires, pour l'enseignement primaire et secondaire. Encore plus mince est le choix des éditions ou traductions des autres textes, où se distinguent seules, un peu plus fréquentes, les Héroïdes. En dehors de la déshérence du goût pour la culture classique, doit-on voir dans cette maltraitance persistante de l'auteur une pure décence de façade, et l'hypocrisie d'une éducation policée qui cède aux conventions sans se priver de la possibilité du libertinage? Ou héritons-nous de vieilles habitudes scolaires, réticentes à ce qui embarrasse les pédagogues? L’un et l'autre sans doute, et pour longtemps

30 PubliiNasonis Ouidiiopera [...], Lyon, Anisson, Posuel et Rigaud, 1689. Voir H. CasanovaRobin, “ Ovide », dans M. Furno (éd.), La collection Ad Vsum Delphini, vol. 2, Grenoble, 2005, p. 455-464. 
probablement, car l'embarras nourrit la mise à l'écart, qui elle-même nourrit la curiosité, apanage seulement de quelques lecteurs audacieux.

\section{Martine Furno}

IHRIM, UMR 5317, Ens Lyon

Université Grenoble Alpes

87 avenue de Romans

26000 Valence

martine.furno@ens-lyon.fr 
ANABASES

Traditions et Réceptions de l'Antiquité

Revue de l'équipe de recherche E.R.A.S.M.E.

Université Toulouse-Jean Jaurès (UT2J)

NoRMES RÉDACTIONNELLES

ANABASES publie des articles dans cinq langues : français, anglais, allemand, italien et espagnol.

Les articles ne dépasseront pas 35000 signes et seront conformes aux normes de la revue,

disponibles sur le site web : http://plh.univ-tlse2.fr

Les articles seront pourvus d'un résumé en français et en anglais, ainsi que de six à huit mots-clés dans ces deux langues.

Les articles pourront être accompagnés de planches en noir et blanc.

Les comptes rendus compteront de 4500 à 6000 signes.

Site web avec Présentation, Sommaire de tous les Numéros et Bulletin d’Abonnement

http://plh.univ-tlse2.fr

Revues.org : http://anabases.revues.org

\section{Courrier}

Pour les articles :

Clément Bur (clement.bur@univ-jfc.fr)

Catherine VALENTI (catherine.valenti@univ-tlse2.fr)

Pour les comptes rendus :

NoémieVILlacÈQue (noemie.villaceque@univ-reims.fr)

Université Toulouse-Jean Jaurès (UT2J)

Équipe P.L.H. - E.R.A.S.M.E. (EA4601)

Maison de la recherche (MdR)

5, allées Antonio Machado

F-31058 Toulouse Cedex 9

Tél. : 0033/(0)5.61.50.25.56 et 57

Fax : 0033/(0)5.61.50.24.90 


\section{Historiographie et identités culturelles}

Carole QuATRELIVRe, Le sanctuaire gaulois de Gournay-sur-Aronde (Oise). Retour sur une découverte exceptionnelle des années 1970

\section{Traditions du patrimoine antique}

Christophe CorbIER, Le pindarisme et l'archéologie musicale : style, valeur et authenticité de la première Pythique à l'époque moderne

Arnaud AmiLien, Hélène en Égypte : Hérodote en dialogue avec l'épopée

\section{Archéologie des savoirs}

Sébastien CAzalas, Au jardin des exempla. Rhétorique et stratégie de l'exemplum antique dans l'œuvre politique de Jean Juvénal des Ursins (1388-1473)

Dossier - Perpétuer Ovide : aspects moraux, éditoriaux, linguistiques et culturels $\left(\mathrm{xIV}^{\mathrm{e}}-\mathrm{XvIII}{ }^{\mathrm{e}} \mathrm{s}\right.$.)

Francesca DelL'Oro, Introduction

Hélène Casanova-Robin, L'audace châtiée : Phaéton, Actéon et Icare dans la tradition latine jusqu'à la Renaissance, tours et détours d'un symbolisme

Dylan Bovet, Le commentaire latin des Métamorphoses d'Ovide : pratiques humanistes et évolutions de Regius-Micyllus (1543) à Burmann-Heinsius (I727)

Martine Furno, Ovide en classe, ou un auteur en éclats

Basil Neurs, D'un Ovide chrétien à un Ovide burlesque, du Moyen Âge au Grand Siècle : continuités et changements dans la traduction et dans l'illustration des Métamorphoses perçus à travers deux éditions du xvir siècle

Olivier ThÉvenaz, Épilogue

\section{Actualités et débats}

Tiphaine-Annabelle BESNARD, 2019 : l'année pop des musées d'archéologie. Retour sur l'exposition romaine Il classico si fa pop. Di scavi, copie e altri pasticci

\section{Relire les classiques des sciences de l'Antiquité}

Jean-Pierre AıBERT, Le premier Detienne : une relecture de « La notion mythique d'A $\lambda \hat{\eta} \theta \varepsilon\llcorner\alpha$ » (REG, 1960, p. 27-35)

Marcel DetIENNE, La notion mythique d'A $\lambda \hat{\eta} \theta \varepsilon \varepsilon \alpha$

\section{L'atelier de l'histoire : chantiers historiographiques}

Antiquités numériques (coordonné par Élodie Guillon) (1)

Élodie GuILLON, Introduction

Jaime ALvar, Le projet EPIDI : Epítetos divinos. Experiencia religiosay relaciones de poder en Hispania

Les mots de l'Antiquité (coordonné par Magali Soulatges) (10)

Jack Tномаs, L’Antiquité dans les toponymes de l’État de New York

Actualité du théâtre (coordonné par Malika Bastin-Hammou) (4)

Mathieu Ferrand, “Avons-nous perdu le Soleil ? / Ou l'avons-nous chassé ? ”

Thyeste de Sénèque, traduit par Florence Dupont. Mise en scène de

Thomas Jolly (Avignon, 2018).

Voyages et Voyageurs (coordonné parVéronique Krings) (11)

Claude Azıza, Freud à Pompéi

\section{Comptes rendus de lecture}

